OPEN ACCESS

Edited by:

Liang Cheng,

Harbin Medical University, China

Reviewed by:

Hua Tang,

Southwest Medical University, China

Xiaofeng Song,

Nanjing University of Aeronautics and

Astronautics, China

*Correspondence:

Lijun Zhang

c7zlj@szpt.edu.cn

Xi Su

xisu_fsfy@163.com

Specialty section:

This article was submitted to

Molecular and Cellular Pathology,

a section of the journal

Frontiers in Cell and Developmental

Biology

Received: 30 December 2021 Accepted: 17 January 2022

Published: 01 February 2022

Citation:

Chen Z, Jiao S, Zhao D, Zou Q, Xu L,

Zhang $L$ and Su $X$ (2022) The

Characterization of Structure and

Prediction for Aquaporin in Tumour

Progression by Machine Learning.

Front. Cell Dev. Biol. 10:845622.

doi: $10.3389 /$ fcell.2022.845622

\section{The Characterization of Structure and Prediction for Aquaporin in Tumour Progression by Machine Learning}

\author{
Zheng Chen ${ }^{1,2}$, Shihu Jiao ${ }^{3}$, Da Zhao ${ }^{1,2}$, Quan Zou ${ }^{2,3}$, Lei Xu ${ }^{4}$, Lijun Zhang ${ }^{1 *}$ and Xi Su ${ }^{5 *}$ \\ ${ }^{1}$ School of Applied Chemistry and Biological Technology, Shenzhen Polytechnic, Shenzhen, China, ${ }^{2}$ Institute of Fundamental and \\ Frontier Sciences, University of Electronic Science and Technology of China, Chengdu, China, ${ }^{3}$ Yangtze Delta Region Institute \\ (Quzhou), University of Electronic Science and Technology of China, Quzhou, China, ${ }^{4}$ School of Electronic and Communication \\ Engineering, Shenzhen Polytechnic, Shenzhen, China, ${ }^{5}$ Foshan Maternal and Child Health Hospital, Foshan, China
}

Recurrence and new cases of cancer constitute a challenging human health problem. Aquaporins (AQPS) can be expressed in many types of tumours, including the brain, breast, pancreas, colon, skin, ovaries, and lungs, and the histological grade of cancer is positively correlated with AQP expression. Therefore, the identification of aquaporins is an area to explore. Computational tools play an important role in aquaporin identification. In this research, we propose reliable, accurate and automated sequence predictor IAQPs-RF to identify AQPs. In this study, the feature extraction method was 188D (global protein sequence descriptor, GPSD). Six common classifiers, including random forest (RF), NaiveBayes (NB), support vector machine (SVM), XGBoost, logistic regression (LR) and decision tree (DT), were used for AQP classification. The classification results show that the random forest (RF) algorithm is the most suitable machine learning algorithm, and the accuracy was $97.689 \%$. Analysis of Variance (ANOVA) was used to analyse these characteristics. Feature rank based on the ANOVA method and IFS strategy was applied to search for the optimal features. The classification results suggest that the 26th feature (neutral/hydrophobic) and 21st feature (hydrophobic) are the two most powerful and informative features that distinguish AQPs from non-AQPs. Previous studies reported that plasma membrane proteins have hydrophobic characteristics. Aquaporin subcellular localization prediction showed that all aquaporins were plasma membrane proteins with highly conserved transmembrane structures. In addition, the 3D structure of aquaporins was consistent with the localization results. Therefore, these studies confirmed that aquaporins possess hydrophobic properties. Although aquaporins are highly conserved transmembrane structures, the phylogenetic tree shows the diversity of aquaporins during evolution. The PCA showed that positive and negative samples were well separated by 54D features, indicating that the 54D feature can effectively classify aquaporins. The online prediction server is accessible at http://lab.malab.cn/ acy/iAQP.

Keywords: cancer, random forest, anova, 3D structure, machine learning 


\section{INTRODUCTION}

Water, as one of the most widely existing molecules, is the basic requirement for the development of organisms. Aquaporins (AQPs) are a large and evolutionarily conserved family of proteins that facilitate water absorption and flow across cytoplasmic compartments and cell membranes in microorganisms, animals, and plants. From a previous study, aquaporins, as water channel proteins, not only take part in water molecule transport but also respond to other small molecule transport, such as glycerol, urea, ammonia, and $\mathrm{CO}_{2}$, which help those molecules cross cell membranes (Preston et al., 1992; Ma et al., 1997; Agre et al., 2002; Nielsen et al., 2002; Rojek et al., 2008). In the aquaporin family, some aquaporins are primarily water selective, such as AQP1, AQP2, AQP4, AQP5 and AQP8, while other parts of the aquaporins, such as $\mathrm{AQP} 3, \mathrm{AQP7}, \mathrm{AQP} 9$, and $\mathrm{AQP} 10$, transport water, glycerol and other small solutes (Verkman, 2005). Aquaporins are small highly conserved membrane proteins that can selectively promote water molecule transportation through the cell membrane. Aquaporins (AQPs), with a molecular weight of $28 \mathrm{kDa}$, were first found in the membrane of human red blood cells (Agre et al., 2002). AQPs usually exist as tetramers; when water passes through these narrow channels, the conformation of AQPs can decide whether water passes through the cell membrane.

AQPs not only act as channels to take part in water and small molecule transport but are also widely related to a variety of pathophysiological statuses in cells. Evidence of AQPs in cell proliferation has aroused great interest in the research of AQPs in tumour progression (Levin and Verkman, 2006; Zhang et al., 2010; Jung et al., 2011; Nakahigashi et al., 2011; Di Giusto et al., 2012; Direito et al., 2016; De Ieso and Yool, 2018). At present, AQPs can be expressed in many types of tumours, including in the brain (Maugeri et al., 2016; Lan et al., 2017), breast (Jung et al., 2011), pancreas (Arsenijevic et al., 2019), colon (Nagaraju et al., 2016), skin (Hara-Chikuma and Verkman, 2008a), ovaries (Kasa et al., 2019) and lung (Chae et al., 2008). There was a positive correlation between the histological tumour grade and AQP expression, such as the expression of AQP4 in diffuse astrocytoma (Saadoun et al., 2002a; Kröger et al., 2004).

For colorectal cancer, the expression of AQP8 decreased (Fischer et al., 2001), while that of AQP1, AQP3 and AQP5 increased (Moon et al., 2003), indicating that AQPs can be expressed in tumours in humans. In general, AQP expression is upregulated in tumours. Therefore, many studies speculate that aquaporins allow water to penetrate, resulting in rapid tumour mass formation. In astrocytomas, the expression level of AQP4 is related to the amount of oedema but not to survival status (Saadoun et al., 2002a; Warth et al., 2007). Recent studies have indicated that $\mathrm{AQPs}$, as prognostic markers, have a potential role in tumour-associated oedema because they participate in angiogenesis, tumour cell migration and proliferation (Saadoun et al., 2005a; Saadoun et al., 2005b; Hara-Chikuma and Verkman, 2006; Auguste et al., 2007; Hara-Chikuma and Verkman, 2008b). AQP1, AQP4 and AQP9 are expressed in brain tumours, and AQP4 expression increases with the severity of brain oedema (Saadoun et al., 2002a; Ding et al., 2010; Wang and
Owler, 2011; Ding et al., 2013; Maugeri et al., 2016; Lan et al., 2017). In brain, lung, prostate and colon tumours, AQP1 with high expression participates in cell migration and tumour angiogenesis (Saadoun et al., 2002b; Saadoun et al., 2005b; Mobasheri et al., 2005; Kang et al., 2008). AQP3 has increased expression in ESCA, COAD, LUAD and LIHC (Marlar et al., 2017). AQP3 knockout mice can inhibit the development of skin tumours, and tumorigenesis can utilize ATP produced by AQP3mediated glycerol transport (Hara-Chikuma and Verkman, 2008a). AQP5 is also related to the migration, metastasis, and poor prognosis of cancer cells in BRCA (breast cancer) (Jung et al., 2011; Lee et al., 2014; Jensen et al., 2016). AQP5-regulating miRNAs inhibit BRCA cell migration through exosomemediated delivery (Park et al., 2020). Under exosomemediated delivery, AQP5-regulated miRNAs inhibit BRCA cell migration (Park et al., 2020).

Aquaporins play a role in the development and prognosis of various cancers, so the machine learning recognition method of aquaporins is also one of the hot spots in cancer research. Machine learning methods are applied to establish a novel and efficient classification model of aquaporins and are helpful to accelerate the recognition of aquaporins. The amino acid sequence composition of the protein is considered to be a sequence feature of the protein (Tyagi et al., 2013).

There are two methods for protein classification methods, as follows: one is based on protein sequence information (Liu et al., 2020a; Zhang et al., 2021), and the other is based on protein structure features (Liu et al., 2019; Cai et al., 2020). The sequencebased protein classification method extracts features by using the amino acid composition, amino acid number and other sequence information of the protein sequence (Liu et al., 2014). These methods are efficient and useful in predicting a large number of protein sequence datasets (Lou et al., 2014). At present, there are various studies on the classification of protein sequences, such as using logistic regression and support vector machine (SVM) methods to predict DNA binding proteins (Shen and Zou, 2020; Liu et al., 2021a) by considering amino acid proportions, amino acid compositions, amino acid spatial asymmetric distributions and biological coding characteristics of evolutionary information (Szilágyi and Skolnick, 2006; Kumar et al., 2007). The protein classification method based on protein structure identifies proteins by using structure and sequence information (Liu et al., 2014). Previous studies have focused on positive electrostatic potential, protein surface, overall charge and positive patches (Shanahan et al., 2004; Bhardwaj et al., 2005), which have achieved excellent results. Under certain conditions, the prediction accuracy of three protein motifs (helix turning helix, helix hairpin helix, and helix loop helix) is $91.1 \%$, which indicates that this method is efficient for protein determination (Cai et al., 2009).

In our work, to promote the rapid application of AQPs in cancer treatment, a powerful sequence-based analysis method to distinguish the AQPs and cross validation was applied for results demonstration (Figure 1). It is important to develop an effective model to predict AQPs. We propose a sequence-based AQP prediction model that performs stably on various classifiers. The AQP classification model uses the 188D feature extraction 


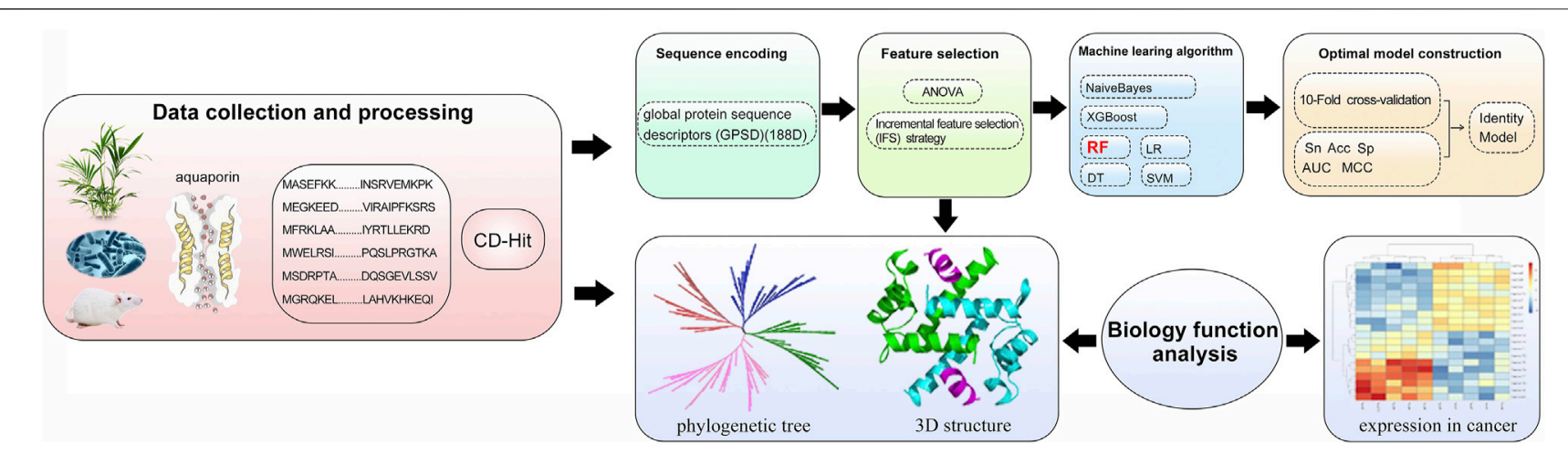

FIGURE 1 | The whole framework of the method iAQPs-RF to identify the aquaporins.

method, applies ANOVA to reduce the dimensionality, and uses different algorithms to optimize the AQP classification model. $188 \mathrm{D}$ is a characteristic of the frequency of continuous amino acid residues in proteins. ANOVA is used to prune features without affecting the accuracy of the predictor.

\section{MATERIALS AND METHODS}

\section{Dataset}

A high-quality dataset is essential for reliable and accurate predictor building (Su et al., 2021). Aquaporin was taken as the positive sample, and the protein sequence was collected from the protein database of the UniProt website (https://www.uniprot.org/) (Chen et al., 2016). Negative samples such as nonaquaporins were extracted from the Pfam database (http://pfam.xfam.org/). To ensure the reliability of the aquaporin dataset, we applied the following criteria to optimize the data: first, the sequences annotated as "prediction" were eliminated; second, we deleted the sequences of other protein fragments; through screening steps, 239 aquaporin sequences and 10,713 nonaquaporin sequences were obtained; third, the CD-HIT program (Fu et al., 2012) was used to eliminate redundant sequences and to avoid overestimating the prediction model (Zou et al., 2020). The cut-off of sequence identity is set to $90 \%$. Finally, 151 aquaporins and 8,994 nonaquaporins were obtained to form the final dataset.

\section{Features Extraction}

One of the main factors for the performance accuracy of the prediction model is the quality of sample feature extraction. The prediction of the protein model mainly depends on the coding strategy of the protein sequence. According to the coding strategy of the protein sequence, the amino acid sequence can be transformed into a numerical vector (Liu et al., 2019; Muhammod et al., 2019; Zhu et al., 2019; Chen et al., 2020; Fu et al., 2020; Tang et al., 2020; Wang et al., 2020; Shao et al., 2021). In this paper, the global protein sequence descriptor (GPSD) method was used to represent the amino acid sequence. Global protein sequence descriptor (GPSD), known as 188 days method. This method mainly converts the sequence into a numerical vector according to the amino acid properties in the protein sequence and generates 188 features. These $188 \mathrm{D}$ features contain the information and properties of amino acid sequences [48,49]. According to the description of the GPSD method, the 188D features can be divided into two parts. The first part is the composition of amino acids. The first $20 \mathrm{D}$ features were obtained by calculating the frequency of amino acids in the protein sequence. The second part is to calculate the physicochemical properties of amino acids, which constitute 168 characteristics. Previous studies have provided detailed information on the eight physicochemical properties of amino acids (Lin et al., 2013; Liu et al., 2018; Li et al., 2019a). The protein sequence was encoded by CTD (C: composition, t: transition, D: distribution) mode to generate $21 \mathrm{D}$ features. Three groups were generated for 20 amino acids for each property. $\mathrm{C}$ is the occurrence frequencies $(1 \times 3 \mathrm{D}=3 \mathrm{D})$. $\mathrm{T}$ is the transition frequency $(1 \times 3 \mathrm{D}=$ $3 \mathrm{D})$. $\mathrm{D}$ is the first, $25,50,75 \%$ and last position of a certain group in the peptide sequence $(5 \times 3=15 \mathrm{D})$. Therefore, $8 *(3+3+15)=$ 168 features were produced for the CTD model.

\section{Classifier}

To find the most suitable machine learning algorithm, six commonly used classifiers are applied, including random forest (RF) (Ru et al., 2019), NaiveBayes, support vector machine (SVM) (Wei et al., 2018a; Dao et al., 2020a; Dao et al., 2020b; Wei et al., 2020), XGBoost (Yu et al., 2021a; Yang et al., 2021), logistic regression (LR) and decision tree (DT) (Li et al., 2019b). These efficient machine learning algorithms are usually used for feature analysis.

\section{Feature Selection}

For machine learning model building, features extracted from sequences always contain noise. A feature selection strategy to solve the information redundancy and overfitting problem can improve the feature representation ability ( $\mathrm{He}$ et al., 2021). Analysis of variance (ANOVA) (Blanca et al., 2017; Wei et al., 2018b; Tang et al., 2018; Su et al., 2019a; Jung et al., 2019; Su et al., 2020; Liu et al., 2021b; Jin et al., 2021) has been used to analyse these characteristics and has been widely used in RNA, DNA and protein prediction. In this study, ANOVA is used to select the optimal features for model training. The feature subset with low redundancy is selected by ANOVA. We sort the original features 
based on the ANOVA feature sorting algorithm and apply the IFS strategy to search the optimal feature subset.

\section{Performance Standard}

To evaluate the prediction accuracy of the model, the data of the following four formulas are usually used to solve the problem of classification prediction.

$$
\begin{gathered}
A c c=\frac{T P+T N}{(T P+T N+F P+F N)} \\
S n=\frac{T P}{(T P+F N)} \\
S p=\frac{T N}{(T N+F P)} \\
M C C=\frac{T P \times T N-F P \times F N}{\sqrt{(T P+F P)(T P+F N)(T N+F P)(T N+F N)}}
\end{gathered}
$$

Accuracy (Acc), specificity (Sp), sensitivity (Sn) and Matthew correlation coefficient (MCC) were the commonly used evaluation parameters (Jiang et al., 2013; Wei et al., 2014; Wei et al., 2017a; Wei et al., 2017b; Wei et al., 2017c; Manavalan et al., 2019a; Manavalan et al., 2019b; Su et al., 2019b; Hong et al., 2019; Zeng et al., 2019; Zhang et al., 2020a; Liu et al., 2020b; Zeng et al., 2020; Yu et al., 2021b; Jin et al., 2021; Shao and Liu, 2021; Zhu et al., 2021). In the formulas, TP was the true positive number, TN was the true negative number, FP was the false-positive number and $\mathrm{FN}$ was the false negative number.

A receiver operating characteristic (ROC) curve was applied to study the prediction performance of the model. The area under the ROC curve (AUC) was used to assess the prediction performance of the model. AUC values of 0.5 and one represent random and perfect models, respectively (Zeng et al., 2017; Zeng et al., 2018; Dao et al., 2019; Feng et al., 2019; Lai et al., 2019; Lin et al., 2019; Zhu et al., 2019; Zhang et al., 2020b; Charoenkwan et al., 2020; Ding et al., 2020a; Ding et al., 2020b; Hasan et al., 2020; Huang et al., 2020; Jin et al., 2020; Li et al., 2020; Wang et al., 2020; Wu and Yu, 2021).

\section{Construction of 3D Structure for AQPs}

To verify the localization of aquaporins, the website (http://www. csbio.situ.edu.cn/bioinf/Cell-PLoc-2/) of the protein localization website and transmembrane prediction website (https://www. novopro.cn/tools/tmhmm.html) were applied to predict the subcellular localization and transmembrane structure of aquaporins. At the same time, Phyre2 software (http://www.sbg. bio.ic.ac.uk/phyre2/html/page.cgi?id=index) was applied for the $3 \mathrm{D}$ structure prediction of aquaporins. The prediction results were visualized by PyMOL (version 2.5.1) software (https://pymol.org/2/).

\section{Construction of Aquaporins Phylogenetic Tree}

The phylogenetic tree of aquaporins was constructed to analyse the evolutionary diversity of the protein. Aquaporin sequence alignment results were analysed by MAFFT online software (https://mafft.cbrc.jp/alignment/server/) and used to construct a phylogenetic tree using IQ-TREE software (multicore version 1.

\begin{tabular}{|c|c|c|c|c|c|}
\hline 188D_P: N = 1:1 & Sn & Sp & Acc & MCC & AUROC \\
\hline XGBoost & 98 & 96.04 & 97.033 & 0.9416 & 0.9949 \\
\hline NaiveBayes & 96.666 & 96.041 & 96.366 & 0.9279 & 0.9763 \\
\hline LR & 97.999 & 94.748 & 96.377 & 0.9289 & 0.9857 \\
\hline DecisionTree & 95.999 & 95.374 & 95.689 & 0.9155 & 0.9569 \\
\hline $\mathrm{RF}$ & 98.666 & 96.707 & 97.689 & 0.9544 & 0.9987 \\
\hline SVM & 95.332 & 96.04 & 95.7 & 0.9153 & 0.9917 \\
\hline 188D_P: $N=1: 2$ & Sn & Sp & Acc & MCC & AUROC \\
\hline NaiveBayes & 98 & 97.667 & 97.793 & 0.9531 & 0.9765 \\
\hline SVM & 92.75 & 98.334 & 96.471 & 0.9224 & 0.9965 \\
\hline LR & 96.083 & 98.001 & 97.359 & 0.9425 & 0.9958 \\
\hline $\mathrm{RF}$ & 97.332 & 98.344 & 98.012 & 0.9564 & 0.9978 \\
\hline XGBoost & 96.708 & 96.677 & 96.682 & 0.9284 & 0.9954 \\
\hline DecisionTree & 92.041 & 93.687 & 93.141 & 0.8518 & 0.9286 \\
\hline 188D_P: $N=1: 3$ & Sn & Sp & Acc & MCC & AUROC \\
\hline NaiveBayes & 97.333 & 96.015 & 96.357 & 0.9102 & 0.9765 \\
\hline SVM & 90.75 & 99.334 & 97.181 & 0.9244 & 0.9979 \\
\hline LR & 95.417 & 98.445 & 97.682 & 0.9394 & 0.9952 \\
\hline $\mathrm{RF}$ & 96.666 & 98.455 & 98.013 & 0.948 & 0.9979 \\
\hline XGBoost & 95.374 & 98.011 & 97.343 & 0.9306 & 0.995 \\
\hline DecisionTree & 93.999 & 96.697 & 96.024 & 0.8974 & 0.9535 \\
\hline 188D_P: $N=1: 4$ & Sn & Sp & Acc & MCC & AUROC \\
\hline NaiveBayes & 97.333 & 97.18 & 97.22 & 0.9206 & 0.9771 \\
\hline SVM & 92.083 & 99.005 & 97.617 & 0.9256 & 0.9946 \\
\hline LR & 93.457 & 97.844 & 96.953 & 0.9074 & 0.9942 \\
\hline $\mathrm{RF}$ & 94.709 & 99.166 & 98.274 & 0.9461 & 0.9967 \\
\hline XGBoost & 95.333 & 98.668 & 98.007 & 0.9391 & 0.9958 \\
\hline DecisionTree & 92.708 & 98.841 & 97.614 & 0.9248 & 0.9578 \\
\hline 188D_P: $N=1: 5$ & Sn & Sp & Acc & $\mathrm{MCC}$ & AUROC \\
\hline NaiveBayes & 96.666 & 97.084 & 97.019 & 0.9022 & 0.9773 \\
\hline SVM & 92.083 & 99.205 & 98.015 & 0.9292 & 0.9954 \\
\hline LR & 93.999 & 98.143 & 97.46 & 0.9136 & 0.996 \\
\hline $\mathrm{RF}$ & 94.667 & 99.338 & 98.562 & 0.9486 & 0.9975 \\
\hline XGBoost & 95.333 & 98.94 & 98.341 & 0.9414 & 0.9963 \\
\hline DecisionTree & 91.374 & 98.01 & 96.905 & 0.8924 & 0.9469 \\
\hline 188D_P: N (151:8,994) & Sn & Sp & Acc & MCC & AUROC \\
\hline XGBoost & 86.084 & 99.934 & 99.703 & 0.9062 & 0.9989 \\
\hline NaiveBayes & 96.666 & 97.977 & 97.955 & 0.6522 & 0.9793 \\
\hline LR & 84.082 & 99.635 & 99.374 & 0.8158 & 0.9975 \\
\hline DecisionTree & 72.208 & 99.365 & 98.918 & 0.6827 & 0.8579 \\
\hline $\mathrm{RF}$ & 82.75 & 99.912 & 99.626 & 0.879 & 0.995 \\
\hline SVM & 31.167 & 100 & 98.866 & 0.5503 & 0.9916 \\
\hline
\end{tabular}
6.12). The best fitting model for the phylogenetic tree was $L G+F$
TABLE 1 | Preliminary results of different feature descriptors using different classifiers.

+ R6 (Kalyaanamoorthy et al., 2017). The ultrafast bootstrap method was used for phylogenetic assessment, and 1,000 replicates per method were chosen in this work (Guindon et al., 2010; Minh et al., 2013; Hoang et al., 2018). The tree file was visualized by the iTOL website (https://itol.embl.de/).

\section{EXPERIMENT}

\section{Performance of Features Based on the 188-Dimensional Method (GPSD)}

To select the best classifier for the AQP sequences, six widely used machine learning classifiers were employed to classify the features 


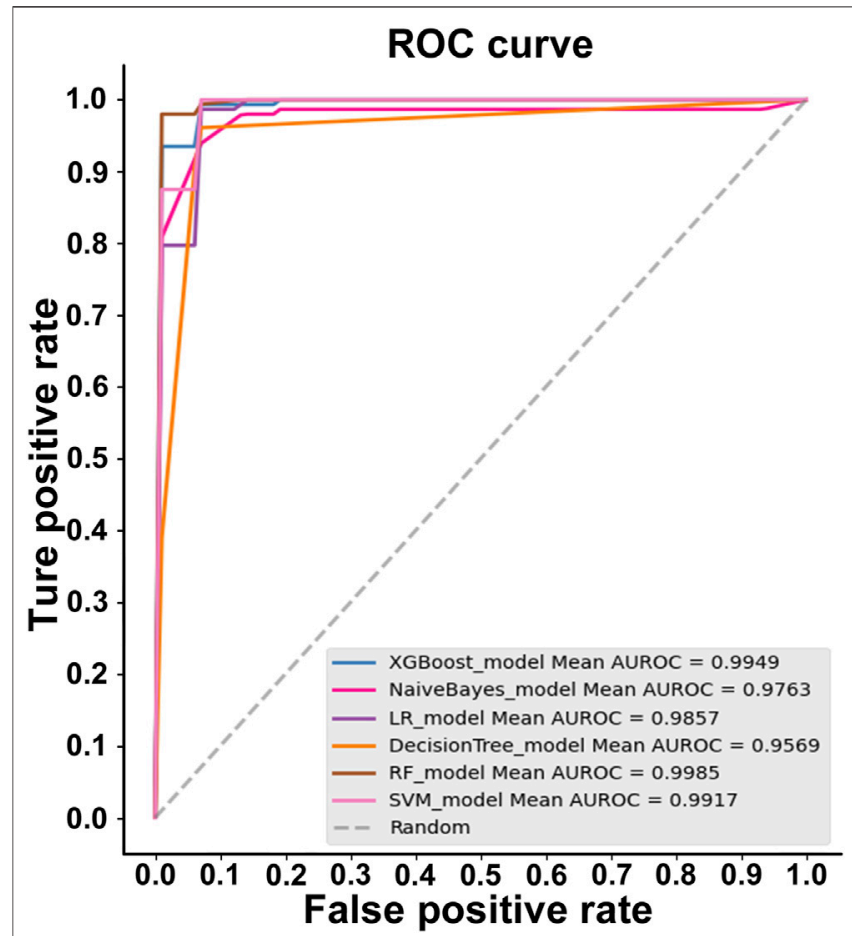

FIGURE 2 | ROC curves for the best performing feature with different classifiers.

of AQP sequences extracted by the 188-dimensional method (GPSD). For feature extraction by the 188-dimensional method (GPSD), we applied different ratios for the number of positive and negative samples $(1: 1,1: 2,1: 3,1: 4,1: 5$, and 151: $8,994)$, and the results were classified by six machine learning classifiers (XGBoost, Naivebayes, LR, decision tree, RF and SVM). The results of all classifiers in the tenfold cross-validation were compared, and the comparison results are shown in Table 1.

The results of Table 1 show that the different proportions of positive and negative samples indicated that $P: N=1: 1$ was the best ratio for the following analysis. Although the values of $1: 2$, 1:3, 1:4, 1:5 and 151:8,989 have higher values in SP and ACC, the values of Sn, MCC and AUROC are lower compared with P: $\mathrm{N}=1: 1$. The increase in negative samples causes data imbalance and overfitting of the model. Therefore, the positive and negative sample ratio column of $\mathrm{P}: \mathrm{N}=1: 1$ is selected for model building.

For the AQP sequences ( $P: N=1: 1)$, random forest $(R F)$ was the best algorithm, with the highest accuracy for the features extracted by the 188-dimensional method (GPSD) (AUC = 0.9987, $\mathrm{Acc}=97.689 \%, \mathrm{MCC}=0.9544, \mathrm{Sn}=98.666 \%, \mathrm{Sp}=$ $96.707 \%)$. XGBoost is the second algorithm with a slightly lower accuracy $(\mathrm{AUC}=0.9949, \mathrm{Acc}=97.033 \%, \mathrm{MCC}=0.9416, \mathrm{Sn}=$ $98 \%, \mathrm{Sp}=96.04 \%)$ compared with the random forest (RF) algorithm. The NaiveBayes, LR, DecisionTree and SVM algorithms have similar accuracies lower than the random forest (RF) algorithm for AQP sequence classification based on the 188-dimensional method (GPSD). The results in Figure 2 indicated that RF was the best classifier with an accuracy of
0.9985, while the other classifiers of XGBoost, Naivebayes, LR, decision tree and SVM had accuracies of 0.9949, 0.9763, 0.9857, 0.9569 and 0.9917 , respectively. In this study, six widely used classifiers are used for classification. The ROC of the RF classifier is 0.9985 , which is relatively high. In general, regarding the evaluated accuracy of the AUC, Acc and MCC values, RF had the best performance in the AQP sequence classification results and was selected as the best classifier for model building.

\section{Effect of Feature Selection Technologies}

However, there are redundant or noisy features among the features extracted by the $188 \mathrm{D}$ method, which will affect the stability of the model. To overcome these effects, we use the ANOVA feature selection method to optimize these features. The optimized classification results of the feature selection method based on ANOVA are shown in Table 2. In addition, the optimal feature $54 \mathrm{D}$ is selected by combining ANOVA with an incremental feature selection (IFS) strategy, as shown in Figure 3A. The comparison results show that the accuracy of the optimal feature selected (ACC $=97.689$ ) is slightly higher than that of the original feature $(\mathrm{ACC}=97.356)$ (Table 2). Therefore, the ANOVA feature selection method was selected for feature optimization.

The PCA method was used to visually analyse the optimal feature (54D) after feature selection by the feature selection method (Figure 3B). Figure 3B indicates that positive and negative samples can almost be separated in the twodimensional visualization diagram, which indicates that the 54D feature can effectively classify AQP proteins.

\section{Feature Distribution Analysis}

In this study, we performed feature analysis after feature selection. By analysing these $188 \mathrm{D}$ features, we determine the attribute information contained in these features. The results of feature analysis are shown in Figure 3C. According to the best feature analysis of the F-score value obtained by ANOVA, the features with an F-score value greater than 100 have a greater contribution to the classification. It can be seen from the figure that among the $188 \mathrm{D}$ features, the first is the 26th dimension feature, which is neutral/hydrophobic, followed by the 21st dimension feature, which is hydrophobic. The 26th dimension feature (neutral/ hydrophobic) and 21st dimension feature (hydrophobic) signs showed that AQPs contained hydrophobic amino acids, which may be associated with the structural and functional properties of AQPs.

\section{Structure Analysis of AQPs}

Through feature selection, we know that hydrophobic features (the 26th dimension feature and 21st dimension feature) are the

TABLE 2 | ANOVA feature selection methods based on random forest.

\begin{tabular}{lccccc}
\hline ANOVA & Sn & Sp & Acc & MCC & AUROC \\
\hline 188D & 98.666 & 96.04 & 97.356 & 0.9479 & 0.9991 \\
ANOVA_54D & 98.666 & 96.707 & 97.689 & 0.9544 & 0.997
\end{tabular}



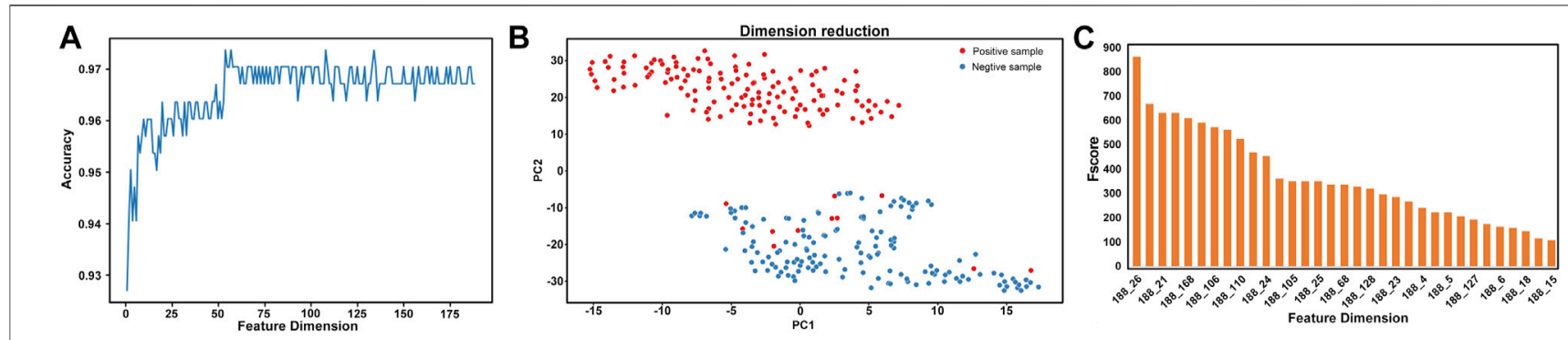

FIGURE 3|Two-step feature selection result display (A) 10-fold CV and independent test accuracy of the RF classifier with the feature number varied (B) dimension reduction results based on the PCA method for the original data with a total of 188 dimensions (C) feature ranking of the F-score method obtained by ANOVA for the data with 188 features.

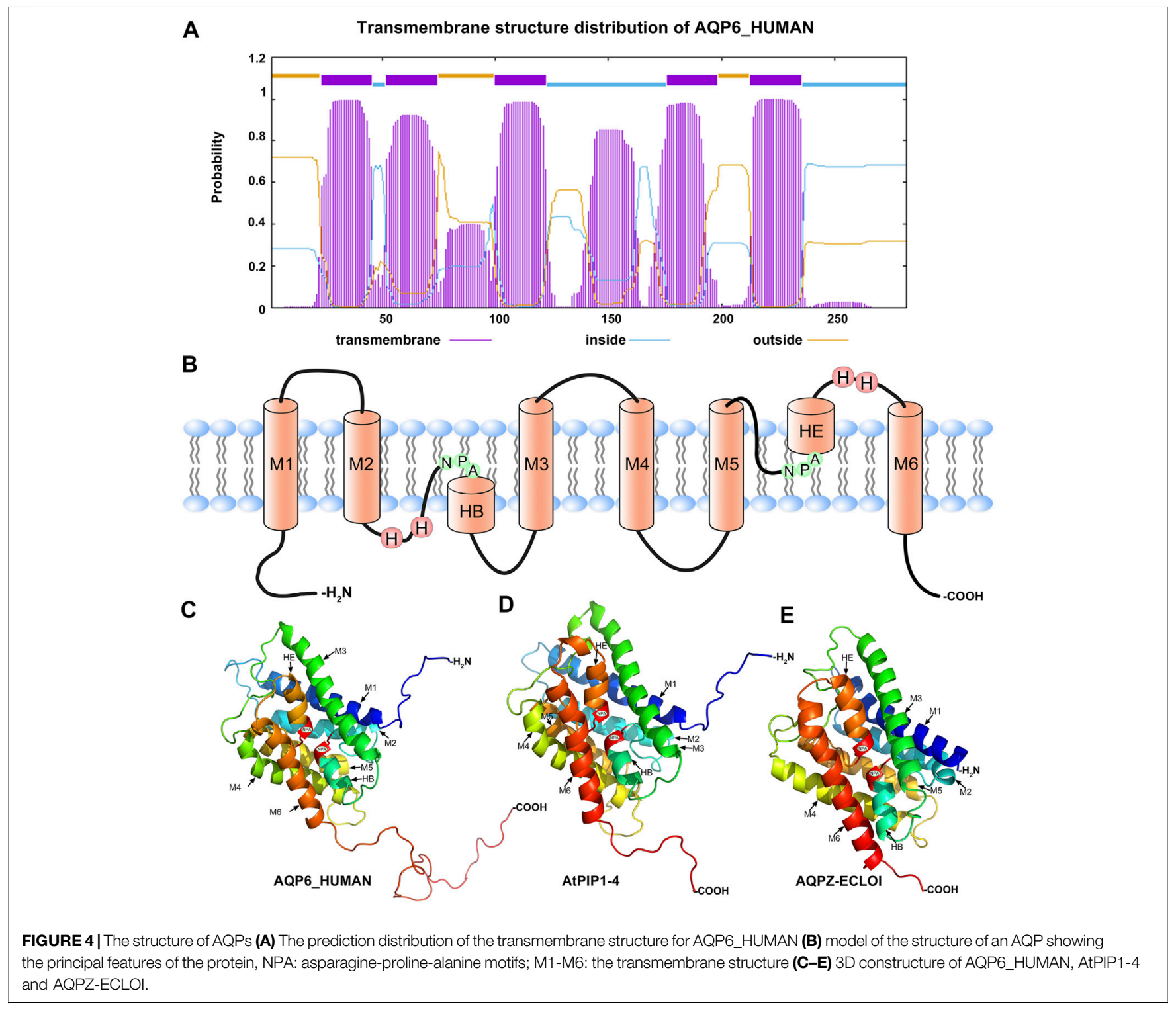




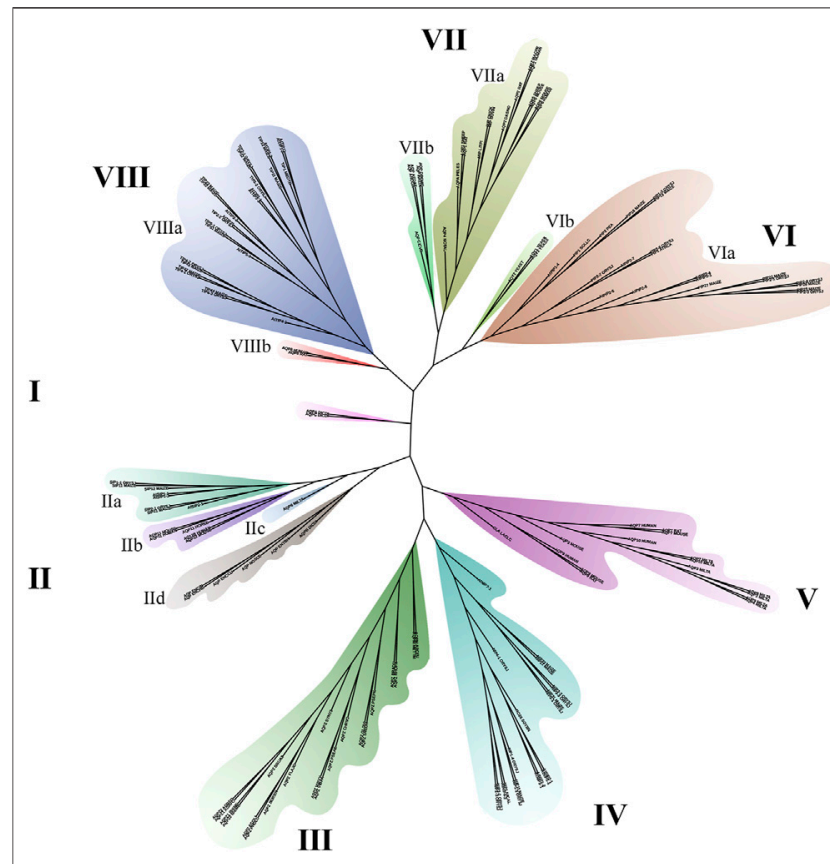

FIGURE 5 | Phylogenetic analysis of positive AQP proteins.

most significant features and make a great contribution to classification. Therefore, we analysed the protein localization of the AQP protein sequence, and the results showed that all AQP proteins were located on the cell membrane (Supplement Table 1). Cells are distinguished by a thin membrane. The core of the membrane is hydrophobic, which means it repels water. Many signals and nutrients cannot pass through the membrane itself but can pass through proteins across the membrane. Membrane proteins are essential for living cells, and plasma membrane proteins also have properties such as hydrophobicity, low solubility and low abundance. Therefore, the enrichment and classification extraction methods of soluble proteins cannot be used for plasma membrane proteins, mainly because the expression level of plasma membrane proteins in cells is very low, and they are highly hydrophobic in nature, which makes them easier to precipitate in aqueous solution and difficult to extract (Luche et al., 2003; Rawlings, 2016).

The Phyre2 website was used to analyse the transmembrane structure of HmAQP7. Figure 2 shows that there are six $\alpha$-helix transmembrane domains (Figure 4A): M1, M2, m3, M4, M5 and M6 (Figure 4B). A six- $\alpha$-helix transmembrane domain forms a pore on the cell membrane to supply water molecules through the cell membrane. When the AQP protein folds, loops $\mathrm{B}(\mathrm{HB})$ and $\mathrm{E}$ (HE), which retain the lipophilic half helix, project to the protein molecular centre, making the highly conserved Asn-Pro-Asp (NPA) motif present the opposite direction, thus regulating the single file conductance of water and acting as a cation and proton exclusion filter (Figures 4B-E).

\section{Evolution and Diversity}

Aquaporin is a conserved membrane protein that contains highly conserved NAP domains and $\alpha$-helical transmembrane domains in bacteria (Figure 4E), plants (Figure 4D) and humans (Figure 4C). To better verify the phylogenetic and evolutionary relationship of AQPs, 151 AQP protein sequences containing human, mouse, insect, fungus and bacteria were applied to construct a phylogenetic tree (Figure 5).

The results indicated that the $151 \mathrm{AQP}$ protein sequences were divided into eight groups (Figure 5). The length of branches indicates the genetic relationship of AQP sequences. Among them, group III and group IV belong to plant and bacteria branches, respectively. Group II is the most complex branch, including the aquaporins of fungi, bacteria and animals. Among them, the VIa and VIIIa branches are plant subfamilies. AQPs of the VIIa and VIIb subfamilies belong to animals and insects, respectively. Group V contains one bacterial AQPZ and 15 animal AQPs, of which 7 belong to Tardigrade.

\section{Expression of AQPs in Tumour Tissue}

AQPs are considered to be important prognostic markers of cancers (Chow et al., 2020), so the expression of AQPs in cancer tissues is also crucial. Figure 6 shows the expression level of AQP transcripts in 33 tumour tissues. AQP1_HUMAN has a high expression level in all tumour tissues and plays an important role in tumour angiogenesis and endothelial cell migration (Saadoun et al., 2005b). AQP3_HUMAN is expressed in almost all tumour tissues except ACC, LGG, UVM and AQP3_HUMAN-mediated glycerol transport, which allows the production of ATP for tumorigenesis. AQP3_HUMAN knockout mice can be resistant to carcinogen induction skin tumours (HaraChikuma and Verkman, 2008a). AQP3_HUMAN and AQP5_HUMAN were also expressed in COAD (Moon et al., 2003), while AQP5_HUMAN expression in human COAD is related to cell proliferation and metastasis. In BRCA, AQP5_HUMAN overexpression is associated with (Jung et al., 2011; Lee et al., 2014; Jensen et al., 2016) migration and poor prognosis in BRCA patients. Consistently, AQP5_HUMAN regulates miRNA migration through exosome-mediated (Park et al., 2020) and inhibits BRCA cell migration. AQP2_HUMAN, AQP12A_HUMAN, AQP12B_HUMAN and MIP had low expression levels in 33 tumour tissues, AQP4_HUMAN was highly expressed in GBM and LGG, and AQP9_HUMAN was highly expressed in LIHC.

\section{Web Server Implementation}

To facilitate the prediction of aquaporins, a user-friendly online server named iAQPs-RF is applied, which can be accessed from http://lab.malab.cn/ acy/iAQP. The protein sequences (FASTA format) were identified to determine whether aquaporins or non-aquaporins use the web server by users. First, the FASTA format protein sequences are enterd or pasted in the left blank box and the submit button is clicked; finally, the results are displayed on the right box. If you want to restart a new task, a clear button or the resubmit button was clicked to clear the sequences in the input box. Finally, new query protein sequences were allowed to enter the input box. The home page provides links of the contact information of authors and relevant data to download. 


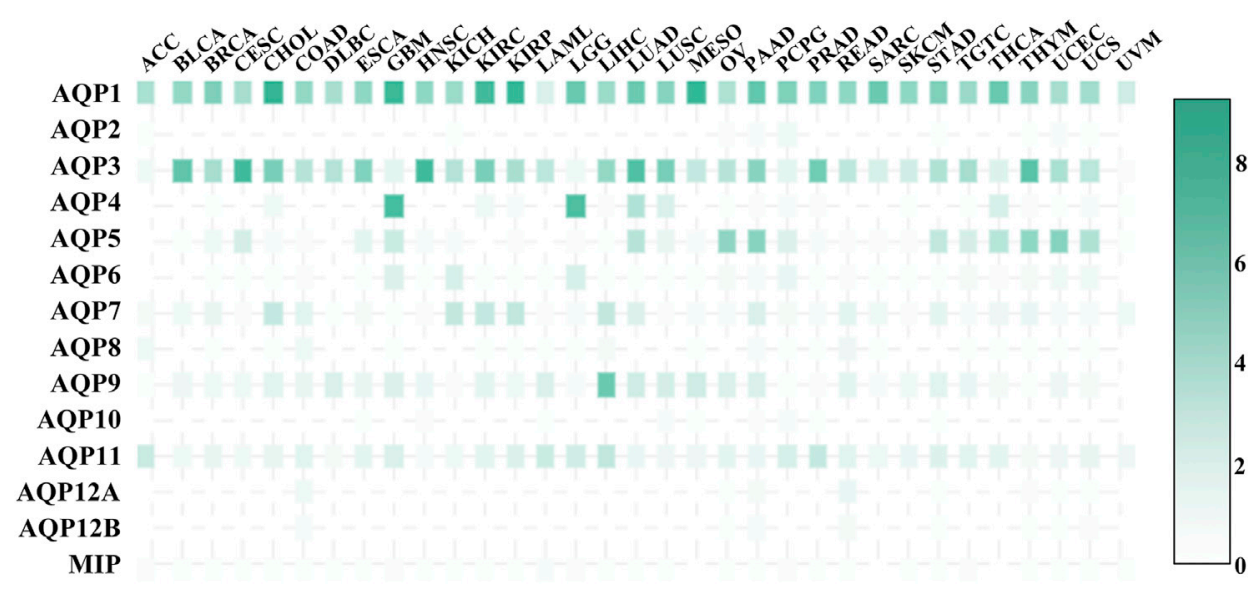

FIGURE 6 | Expression of AQPs in 33 human tumours.

\section{CONCLUSION}

The accurate identification of aquaporins by iAQPs can greatly promote the prediction of aquaporins and research on tumour diseases. In this study, we used the GPSD method to extract protein sequence features and the optimal random forest algorithm to construct new computational aquaporin identifier iAQPs-RF. Combined with the feature selection technique ANOVA, 54 optimal features are selected to build the predictor. According to the F-score value obtained by ANOVA, the 26th dimension feature and 21st dimension feature are ranked as the first and second dimension features among the 188 days features, respectively, and these two features possess neutral/hydrophobic characteristics. These two dimensional features make a great contribution to the classification of aquaporins. At the same time, through the location and 3D structure prediction of aquaporins protein, although the protein divided into eight groups and has diversity in evolution, all the proteins belong to plasma membrane proteins, and the protein sequence contains six $\alpha-$ helix transmembrane domains. The membrane proteins are hydrophobic and contain many hydrophobic amino acids (Luche et al., 2003; Rawlings, 2016), so these results are consistent with aquaporin classification.

The best CV evaluation accuracy of iAQPs-RF was $97.689 \%$. At the same time, a network server is established. iAQPs-RF are expected to be a robust and reliable tool for aquaporin identification. Future work will focus on exploring deep learning to improve the performance of the model.

\section{REFERENCES}

Agre, P., King, L. S., Yasui, M., Guggino, W. B., Ottersen, O. P., Fujiyoshi, Y., et al. (2002). Aquaporin Water Channels - from Atomic Structure to Clinical Medicine. J. Physiol. 542, 3-16. doi:10.1113/jphysiol.2002. 020818

\section{DATA AVAILABILITY STATEMENT}

The original contributions presented in the study are included in the article/Supplementary Material, further inquiries can be directed to the corresponding authors.

\section{AUTHOR CONTRIBUTIONS}

LX, XS and LZ designed the research; ZC and SJ performed the research; $Z C$ and $D Z$ analyzed the data; $Z C$ wrote the manuscript. All authors read and approved the manuscript. The funders had no role in study design, data collection and analysis, decision to publish, or preparation of the manuscript.

\section{FUNDING}

The work was supported by the National Natural Science Foundation of China (No.62002244, No.62001311) and the Post-doctoral Foundation Project of Shenzhen Polytechnic China (No. 6020330002K).

\section{SUPPLEMENTARY MATERIAL}

The Supplementary Material for this article can be found online at: https://www.frontiersin.org/articles/10.3389/fcell.2022.845622/ full\#supplementary-material

Arsenijevic, T., Perret, J., Van Laethem, J.-L., and Delporte, C. (2019). Aquaporins Involvement in Pancreas Physiology and in Pancreatic Diseases. Ijms 20 (20), 5052. doi:10.3390/ijms20205052

Auguste, K. I., Jin, S., Uchida, K., Yan, D., Manley, G. T., Papadopoulos, M. C., et al. (2007). Greatly Impaired Migration of Implanted Aquaporin-4-deficient Astroglial Cells in Mouse Brain toward a Site of Injury. FASEB j. 21 (1), 108-116. doi:10.1096/fj.06-6848com 
Bhardwaj, N., Langlois, R. E., Zhao, G., and Lu, H. (2005). Kernel-based Machine Learning Protocol for Predicting DNA-Binding Proteins. Nucleic Acids Res. 33 (20), 6486-6493. doi:10.1093/nar/gki949

Blanca, M. J., Alarcón, R., Arnau, J., Bono, R., and Bendayan, R. (2017). Nonnormal Data: Is ANOVA Still a Valid Option? Psicothema 29 (4), 552-557. doi:10.7334/psicothema2016.383

Cai, L., Wang, L., Fu, X., Xia, C., Zeng, X., and Zou, Q. (2020). ITP-pred: an Interpretable Method for Predicting, Therapeutic Peptides with Fused Features Low-Dimension Representation. Brief. Bioinform. 22, bbaa367. doi:10.1093/ bib/bbaa367

Cai, Y., He, J., Li, X., Lu, L., Yang, X., Feng, K., et al. (2009). A Novel Computational Approach to Predict Transcription Factor DNA Binding Preference. J. Proteome Res. 8 (2), 999-1003. doi:10.1021/pr800717y

Chae, Y. K., Woo, J., Kim, M.-J., Kang, S. K., Kim, M. S., Lee, J., et al. (2008). Expression of Aquaporin 5 (AQP5) Promotes Tumor Invasion in Human Non Small Cell Lung Cancer. PLoS One 3 (5), e2162. doi:10.1371/journal.pone.0002162

Charoenkwan, P., Yana, J., Schaduangrat, N., Nantasenamat, C., Hasan, M. M., Shoombuatong, W., et al. (2020). iBitter-SCM: Identification and Characterization of Bitter Peptides Using a Scoring Card Method with Propensity Scores of Dipeptides. Genomics 112 (4), 2813-2822. doi:10.1016/ j.ygeno.2020.03.019

Chen, X.-X., Tang, H., Li, W.-C., Wu, H., Chen, W., Ding, H., et al. (2016). Identification of Bacterial Cell Wall Lyases via Pseudo Amino Acid Composition. Biomed. Res. Int. 2016, 1-8. doi:10.1155/2016/1654623

Chen, Z., Zhao, P., Li, F., Marquez-Lago, T. T., Leier, A., Revote, J., et al. (2020). iLearn: an Integrated Platform and Meta-Learner for Feature Engineering, Machine-Learning Analysis and Modeling of DNA, RNA and Protein Sequence Data. Brief. Bioinformatics 21 (3), 1047-1057. doi:10.1093/bib/bbz041

Chow, P. H., Bowen, J., and Yool, A. J. (2020). Combined Systematic Review and Transcriptomic Analyses of Mammalian Aquaporin Classes 1 to 10 as Biomarkers and Prognostic Indicators in Diverse Cancers. Cancers 12, 1911. doi:10.3390/cancers12071911

Dao, F.-Y., Lv, H., Wang, F., Feng, C.-Q., Ding, H., Chen, W., et al. (2019). Identify Origin of Replication in Saccharomyces cerevisiae Using Two-step Feature Selection Technique. Bioinformatics (Oxford, England) 35 (12), 2075-2083. doi:10.1093/bioinformatics/bty943

Dao, F.-Y., Lv, H., Yang, Y.-H., Zulfiqar, H., Gao, H., and Lin, H. (2020). Computational Identification of N6-Methyladenosine Sites in Multiple Tissues of Mammals. Comput. Struct. Biotechnol. J. 18, 1084-1091. doi:10. 1016/j.csbj.2020.04.015

Dao, F.-Y., Lv, H., Zulfiqar, H., Yang, H., Su, W., Gao, H., et al. (2020). A Computational Platform to Identify Origins of Replication Sites in Eukaryotes. Brief Bioinform 22, 1940-1950. doi:10.1093/bib/bbaa017

De Ieso, M. L., and Yool, A. J. (2018). Mechanisms of Aquaporin-Facilitated Cancer Invasion and Metastasis. Front. Chem. 6, 135. doi:10.3389/fchem.2018.00135

Di Giusto, G., Flamenco, P., Rivarola, V., Fernández, J., Melamud, L., Ford, P., et al. (2012). Aquaporin 2-increased Renal Cell Proliferation Is Associated with Cell Volume Regulation. J. Cell. Biochem. 113 (12), 3721-3729. doi:10.1002/jcb.24246

Ding, T., Gu, F., Fu, L., and Ma, Y.-J. (2010). Aquaporin-4 in Glioma Invasion and an Analysis of Molecular Mechanisms. J. Clin. Neurosci. 17 (11), 1359-1361. doi:10.1016/j.jocn.2010.02.014

Ding, T., Zhou, Y., Sun, K., Jiang, W., Li, W., Liu, X., et al. (2013). Knockdown a Water Channel Protein, Aquaporin-4, Induced Glioblastoma Cell Apoptosis. PLoS One 8 (8), e66751. doi:10.1371/journal.pone.0066751

Ding, Y., Tang, J., and Guo, F. (2020a). Identification of Drug-Target Interactions via Dual Laplacian Regularized Least Squares with Multiple Kernel Fusion. Knowledge-Based Syst. 204, 106254. doi:10.1016/j.knosys. 2020.106254

Ding, Y., Tang, J., and Guo, F. (2020b). Identification of Drug-Target Interactions via Fuzzy Bipartite Local Model. Neural Comput. Applic 32, 10303-10319. doi:10.1007/s00521-019-04569-z

Direito, I., Madeira, A., Brito, M. A., and Soveral, G. (2016). Aquaporin-5: from Structure to Function and Dysfunction in Cancer. Cell. Mol. Life Sci. 73 (8), 1623-1640. doi:10.1007/s00018-016-2142-0

Feng, C.-Q., Zhang, Z.-Y., Zhu, X.-J., Lin, Y., Chen, W., Tang, H., et al. (2019). iTerm-PseKNC: a Sequence-Based Tool for Predicting Bacterial Transcriptional Terminators. Bioinformatics (Oxford, England) 35 (9), 1469-1477. doi:10.1093/bioinformatics/bty827
Fischer, H., Stenling, R., Rubio, C., and Lindblom, A. (2001). Differential Expression of Aquaporin 8 in Human Colonic Epithelial Cells and Colorectal Tumors. BMC Physiol. 1, 1. doi:10.1186/1472-6793-1-1

Fu, L., Niu, B., Zhu, Z., Wu, S., Li, W., and Cd-Hit (2012). CD-HIT: Accelerated for Clustering the Next-Generation Sequencing Data. Bioinformatics 28 (23), 3150-3152. doi:10.1093/bioinformatics/bts565

Fu, X., Cai, L., Zeng, X., and Zou, Q. (2020). StackCPPred: a Stacking and Pairwise Energy Content-Based Prediction of Cell-Penetrating Peptides and Their Uptake Efficiency. Bioinformatics 36 (10), 3028-3034. doi:10.1093/ bioinformatics/btaal31

Guindon, S., Dufayard, J.-F., Lefort, V., Anisimova, M., Hordijk, W., and Gascuel, O. (2010). New Algorithms and Methods to Estimate Maximum-Likelihood Phylogenies: Assessing the Performance of PhyML 3.0. Syst. Biol. 59 (3), 307-321. doi:10.1093/sysbio/syq010

Hara-Chikuma, M., and Verkman, A. S. (2006). Aquaporin-1 Facilitates Epithelial Cell Migration in Kidney Proximal Tubule. Jasn 17 (1), 39-45. doi:10.1681/asn. 2005080846

Hara-Chikuma, M., and Verkman, A. S. (2008). Aquaporin-3 Facilitates Epidermal Cell Migration and Proliferation during Wound Healing. J. Mol. Med. 86 (2), 221-231. doi:10.1007/s00109-007-0272-4

Hara-Chikuma, M., and Verkman, A. S. (2008). Prevention of Skin Tumorigenesis and Impairment of Epidermal Cell Proliferation by Targeted Aquaporin-3 Gene Disruption. Mol. Cell Biol 28 (1), 326-332. doi:10.1128/mcb.01482-07

Hasan, M. M., Schaduangrat, N., Basith, S., Lee, G., Shoombuatong, W., and Manavalan, B. (2020). HLPpred-Fuse: Improved and Robust Prediction of Hemolytic Peptide and its Activity by Fusing Multiple Feature Representation. Bioinformatics (Oxford, England) 36 (11), 3350-3356. doi:10.1093/ bioinformatics/btaal60

He, S., Guo, F., Zou, Q., and HuiDing, H. (2021). MRMD2.0: A Python Tool for Machine Learning with Feature Ranking and Reduction. Cbio 15 (10), 1213-1221. doi:10.2174/1574893615999200503030350

Hoang, D. T., Chernomor, O., von Haeseler, A., Minh, B. Q., and Vinh, L. S. (2018), UFBoot2: Improving the Ultrafast Bootstrap Approximation. Mol. Biol. Evol. 35 (2), 518-522. doi:10.1093/molbev/msx281

Hong, Z., Zeng, X., Wei, L., and Liu, X. (2019). Identifying Enhancer-Promoter Interactions with Neural Network Based on Pre-trained DNA Vectors and Attention Mechanism. Bioinformatics 36 (4), 1037-1043. doi:10.1093/ bioinformatics/btz694

Huang, Y., Zhou, D., Wang, Y., Zhang, X., Su, M., Wang, C., et al. (2020). Prediction of Transcription Factors Binding Events Based on Epigenetic Modifications in Different Human Cells. Epigenomics 12 (16), 1443-1456. doi:10.2217/epi-2019-0321

Jensen, H. H., Login, F. H., Koffman, J. S., Kwon, T.-H., and Nejsum, L. N. (2016). The Role of Aquaporin-5 in Cancer Cell Migration: A Potential Active Participant. Int. J. Biochem. Cell Biol. 79, 271-276. doi:10.1016/j.biocel.2016.09.005

Jiang, Q., Wang, G., Jin, S., Li, Y., and Wang, Y. (2013). Predicting Human microRNA-Disease Associations Based on Support Vector Machine. Ijdmb 8 (3), 282-293. doi:10.1504/ijdmb.2013.056078

Jin, Q., Cui, H., Sun, C., Meng, Z., and Su, R. (2021). Free-form Tumor Synthesis in Computed Tomography Images via Richer Generative Adversarial Network. Knowledge-Based Syst. 218, 106753. doi:10.1016/j.knosys.2021.106753

Jin, S., Zeng, X., Xia, F., Huang, W., and Liu, X. (2020). Application of Deep Learning Methods in Biological Networks. Brief Bioinform 22 (2), 1902-1917. doi:10.1093/bib/bbaa043

Jung, H. J., Park, J.-Y., Jeon, H.-S., and Kwon, T.-H. (2011). Aquaporin-5: a Marker Protein for Proliferation and Migration of Human Breast Cancer Cells. PLoS One 6 (12), e28492. doi:10.1371/journal.pone.0028492

Jung, Y., Zhang, H., and Hu, J. (2019). Transformed Low-Rank ANOVA Models for High-Dimensional Variable Selection. Stat. Methods Med. Res. 28 (4), 1230-1246. doi:10.1177/0962280217753726

Kalyaanamoorthy, S., Minh, B. Q., Wong, T. K. F., von Haeseler, A., and Jermiin, L. S. (2017). ModelFinder: Fast Model Selection for Accurate Phylogenetic Estimates. Nat. Methods 14 (6), 587-589. doi:10.1038/nmeth.4285

Kang, S. K., Chae, Y. K., Woo, J., Kim, M. S., Park, J. C., Lee, J., et al. (2008). Role of Human Aquaporin 5 in Colorectal Carcinogenesis. Am. J. Pathol. 173 (2), 518-525. doi:10.2353/ajpath.2008.071198

Kasa, P., Farran, B., Prasad, G. L. V., and Nagaraju, G. P. (2019). Aquaporins in Female Specific Cancers. Gene 700, 60-64. doi:10.1016/j.gene.2019.03.032 
Kröger, S., Wolburg, H., and Warth, A. (2004). Redistribution of Aquaporin-4 in Human Glioblastoma Correlates with Loss of Agrin Immunoreactivity from Brain Capillary Basal Laminae. Acta neuropathologica 107 (4), 311-318. doi:10. 1007/s00401-003-0812-0

Kumar, M., Gromiha, M. M., and Raghava, G. P. (2007). Identification of DNABinding Proteins Using Support Vector Machines and Evolutionary Profiles. BMC Bioinformatics 8, 463. doi:10.1186/1471-2105-8-463

Lai, H.-Y., Zhang, Z.-Y., Su, Z.-D., Su, W., Ding, H., Chen, W., et al. (2019). iProEP: A Computational Predictor for Predicting Promoter. Mol. Ther. - Nucleic Acids 17, 337-346. doi:10.1016/j.omtn.2019.05.028

Lan, Y.-L., Wang, X., Lou, J.-C., Ma, X.-C., and Zhang, B. (2017). The Potential Roles of Aquaporin 4 in Malignant Gliomas. Oncotarget 8 (19), 32345-32355. doi:10.18632/oncotarget.16017

Lee, S. J., Chae, Y. S., Kim, J. G., Kim, W. W., Jung, J. H., Park, H. Y., et al. (2014). AQP5 Expression Predicts Survival in Patients with Early Breast Cancer. Ann. Surg. Oncol. 21 (2), 375-383. doi:10.1245/s10434-013-3317-7

Levin, M. H., and Verkman, A. S. (2006). Aquaporin-3-dependent Cell Migration and Proliferation during Corneal Re-epithelialization. Invest. Ophthalmol. Vis. Sci. 47 (10), 4365-4372. doi:10.1167/iovs.06-0335

Li, J., Pu, Y., Tang, J., Zou, Q., and Guo, F. (2020). DeepATT: a Hybrid Category Attention Neural Network for Identifying Functional Effects of DNA Sequences. Brief. Bioinform. 22, 1. doi:10.1093/bib/bbaa159

Li, M., Xu, H., and Deng, Y. (2019). Evidential Decision Tree Based on Belief Entropy. Entropy 21 (9), 897. doi:10.3390/e21090897

Li, Y., Niu, M., Zou, Q., and Elm-, M. H. C. (2019). ELM-MHC: An Improved MHC Identification Method with Extreme Learning Machine Algorithm. J. Proteome Res. 18 (3), 1392-1401. doi:10.1021/acs.jproteome.9b00012

Lin, C.-W., Chen, P.-N., Chen, M.-K., Yang, W.-E., Tang, C.-H., Yang, S.-F., et al. (2013). Kaempferol Reduces Matrix Metalloproteinase-2 Expression by DownRegulating ERK1/2 and the Activator Protein-1 Signaling Pathways in Oral Cancer Cells. PLoS One 8 (11), e80883. doi:10.1371/journal.pone.0080883

Lin, H., Liang, Z.-Y., Tang, H., and Chen, W. (2019). Identifying Sigma70 Promoters with Novel Pseudo Nucleotide Composition. Ieee/acm Trans. Comput. Biol. Bioinf. 16 (4), 1316-1321. doi:10.1109/tcbb.2017.2666141

Liu, B., Gao, X., and Zhang, H. (2019). BioSeq-Analysis2.0: an Updated Platform for Analyzing DNA, RNA and Protein Sequences at Sequence Level and Residue Level Based on Machine Learning Approaches. Nucleic Acids Res. 47 (20), e127. doi:10.1093/nar/gkz740

Liu, B., Xu, J., Lan, X., Xu, R., Zhou, J., Wang, X., et al. (2014). iDNA-Prot|dis: Identifying DNA-Binding Proteins by Incorporating Amino Acid DistancePairs and Reduced Alphabet Profile into the General Pseudo Amino Acid Composition. PLoS One 9 (9), e106691. doi:10.1371/journal.pone.0106691

Liu, B., Zhu, Y., and Yan, K. (2020). Fold-LTR-TCP: Protein Fold Recognition Based on Triadic Closure Principle. Brief. Bioinform. 21 (6), 2185-2193. doi:10. 1093/bib/bbz139

Liu, J., Su, R., Zhang, J., and Wei, L. (2021). Classification and Gene Selection of Triple-Negative Breast Cancer Subtype Embedding Gene Connectivity Matrix in Deep Neural Network. Brief Bioinform 22, bbaa395. doi:10.1093/bib/bbaa395

Liu, M.-L., Su, W., Wang, J.-S., Yang, Y.-H., Yang, H., and Lin, H. (2020). Predicting Preference of Transcription Factors for Methylated DNA Using Sequence Information. Mol. Ther. - Nucleic Acids 22, 1043-1050. doi:10.1016/j.omtn.2020.07.035

Liu, X.-J., Gong, X.-J., Yu, H., and Xu, J.-H. (2018). A Model Stacking Framework for Identifying DNA Binding Proteins by Orchestrating Multi-View Features and Classifiers. Genes 9 (8), 394. doi:10.3390/genes9080394

Liu, Y., Ouyang, X.-h., Xiao, Z.-X., Zhang, L., and Cao, Y. (2021). A Review on the Methods of Peptide-MHC Binding Prediction. Cbio 15 (8), 878-888. doi:10. 2174/1574893615999200429122801

Lou, W., Wang, X., Chen, F., Chen, Y., Jiang, B., and Zhang, H. (2014). Sequence Based Prediction of DNA-Binding Proteins Based on Hybrid Feature Selection Using Random Forest and Gaussian Naïve Bayes. PLoS One 9 (1), e86703. doi:10.1371/journal.pone.0086703

Luche, S., Santoni, V., and Rabilloud, T. (2003). Evaluation of Nonionic and Zwitterionic Detergents as Membrane Protein Solubilizers in Two-Dimensional Electrophoresis. Proteomics 3 (3), 249-253. doi:10.1002/pmic.200390037

Ma, T., Yang, B., and Verkman, A. S. (1997). Cloning of a Novel Water and UreaPermeable Aquaporin from Mouse Expressed Strongly in colon, Placenta, Liver, and Heart. Biochem. Biophysical Res. Commun. 240 (2), 324-328. doi:10.1006/ bbrc. 1997.7664
Manavalan, B., Basith, S., Shin, T. H., Wei, L., and Lee, G. (2019). mAHTPred: a Sequence-Based Meta-Predictor for Improving the Prediction of Antihypertensive Peptides Using Effective Feature Representation. Bioinformatics 35 (16), 2757-2765. doi:10.1093/bioinformatics/bty1047

Manavalan, B., Basith, S., Shin, T. H., Wei, L., and Lee, G. (2019). Meta-4mCpred: A Sequence-Based Meta-Predictor for Accurate DNA 4mC Site Prediction Using Effective Feature Representation. Mol. Ther. - Nucleic Acids 16, 733-744. doi:10.1016/j.omtn.2019.04.019

Marlar, S., Jensen, H. H., Login, F. H., and Nejsum, L. N. (2017). Aquaporin-3 in Cancer. Ijms 18 (10), 2106. doi:10.3390/ijms18102106

Maugeri, R., Schiera, G., Di Liegro, C., Fricano, A., Iacopino, D., and Di Liegro, I. (2016). Aquaporins and Brain Tumors. Ijms 17 (7), 1029. doi:10.3390/ ijms 17071029

Minh, B. Q., Nguyen, M. A. T., and von Haeseler, A. (2013). Ultrafast Approximation for Phylogenetic Bootstrap. Mol. Biol. Evol. 30 (5), 1188-1195. doi:10.1093/molbev/mst024

Mobasheri, A., Airley, R., Hewitt, S., and Marples, D. (2005). Heterogeneous Expression of the Aquaporin 1 (AQP1) Water Channel in Tumors of the Prostate, Breast, Ovary, colon and Lung: a Study Using High Density Multiple Human Tumor Tissue Microarrays. Int. J. Oncol. 26 (5), 1149-1158. doi:10. 3892/ijo.26.5.1149

Moon, C., Soria, J.-C., Jang, S. J., Lee, J., Hoque, M. O., Sibony, M., et al. (2003). Involvement of Aquaporins in Colorectal Carcinogenesis. Oncogene 22 (43), 6699-6703. doi:10.1038/sj.onc.1206762

Muhammod, R., Ahmed, S., Md Farid, D., Shatabda, S., Sharma, A., and Dehzangi, A. (2019). PyFeat: a Python-Based Effective Feature Generation Tool for DNA, RNA and Protein Sequences. Bioinformatics (Oxford, England) 35 (19), 3831-3833. doi:10.1093/bioinformatics/btz165

Nagaraju, G. P., Basha, R., Rajitha, B., Alese, O. B., Alam, A., Pattnaik, S., et al. (2016). Aquaporins: Their Role in Gastrointestinal Malignancies. Cancer Lett. 373 (1), 12-18. doi:10.1016/j.canlet.2016.01.003

Nakahigashi, K., Kabashima, K., Ikoma, A., Verkman, A. S., Miyachi, Y., and HaraChikuma, M. (2011). Upregulation of Aquaporin-3 Is Involved in Keratinocyte Proliferation and Epidermal Hyperplasia. J. Invest. Dermatol. 131 (4), 865-873. doi:10.1038/jid.2010.395

Nielsen, S., Frøkiær, J., Marples, D., Kwon, T.-H., Agre, P., and Knepper, M. A. (2002). Aquaporins in the Kidney: from Molecules to Medicine. Physiol. Rev. 82 (1), 205-244. doi:10.1152/physrev.00024.2001

Park, E. J., Jung, H. J., Choi, H. J., Jang, H. J., Park, H. J., Nejsum, L. N., et al. (2020). Exosomes Co-expressing AQP5-targeting miRNAs and IL-4 Receptor-binding Peptide Inhibit the Migration of Human Breast Cancer Cells. FASEB j. 34 (2), 3379-3398. doi:10.1096/fj.201902434R

Preston, G. M., Carroll, T. P., Guggino, W. B., and Agre, P. (1992). Appearance of Water Channels in Xenopus Oocytes Expressing Red Cell CHIP28 Protein. Science 256 (5055), 385-387. doi:10.1126/science.256.5055.385

Rawlings, A. E. (2016). Membrane Proteins: Always an Insoluble Problem? Biochem. Soc. Trans. 44, 790-795. doi:10.1042/BST20160025

Rojek, A., Praetorius, J., Frøkiaer, J., Nielsen, S., and Fenton, R. A. (2008). A Current View of the Mammalian Aquaglyceroporins. Annu. Rev. Physiol. 70, 301-327. doi:10.1146/annurev.physiol.70.113006.100452

$\mathrm{Ru}, \mathrm{X}$., Li, L., and Zou, Q. (2019). Incorporating Distance-Based Top-N-Gram and Random Forest to Identify Electron Transport Proteins. J. Proteome Res. 18 (7), 2931-2939. doi:10.1021/acs.jproteome.9b00250

Saadoun, S., Papadopoulos, M. C., Davies, D. C., Krishna, S., and Bell, B. A. (2002). Aquaporin-4 Expression Is Increased in Oedematous Human Brain Tumours. J. Neurol. Neurosurg. Psychiatry 72 (2), 262-265. doi:10.1136/jnnp. 72.2 .262

Saadoun, S., Papadopoulos, M. C., Davies, D. C., Bell, B. A., and Krishna, S. (2002). Increased Aquaporin 1 Water Channel Expression Inhuman Brain Tumours. Br. J. Cancer 87 (6), 621-623. doi:10.1038/sj.bjc.6600512

Saadoun, S., Papadopoulos, M. C., Hara-Chikuma, M., and Verkman, A. S. (2005). Impairment of Angiogenesis and Cell Migration by Targeted Aquaporin-1 Gene Disruption. Nature 434 (7034), 786-792. doi:10.1038/ nature 03460

Saadoun, S., Papadopoulos, M. C., Watanabe, H., Yan, D., Manley, G. T., and Verkman, A. S. (2005). Involvement of Aquaporin-4 in Astroglial Cell Migration and Glial Scar Formation. J. Cel. Sci. 118 (Pt 24), 5691-5698. doi: $10.1242 /$ jcs. 02680 
Shanahan, H. P., Garcia, M. A., Jones, S., and Thornton, J. M. (2004). Identifying DNA-Binding Proteins Using Structural Motifs and the Electrostatic Potential. Nucleic Acids Res. 32 (16), 4732-4741. doi:10.1093/nar/gkh803

Shao, J., and Liu, B. (2021). ProtFold-DFG: Protein Fold Recognition by Combining Directed Fusion Graph and PageRank Algorithm. Brief Bioinform 22 (3), bbaa192. doi:10.1093/bib/bbaa192

Shao, J., Yan, K., and Liu, B. (2021). FoldRec-C2C: Protein Fold Recognition by Combining Cluster-To-Cluster Model and Protein Similarity Network. Brief Bioinform 22 (3), bbaa144. doi:10.1093/bib/bbaa144

Shen, Z., and Zou, Q. (2020). Basic Polar and Hydrophobic Properties Are the Main Characteristics that Affect the Binding of Transcription Factors to Methylation Sites. Bioinformatics 36 (15), 4263-4268. doi:10.1093/bioinformatics/btaa492

Su, R., Hu, J., Zou, Q., Manavalan, B., and Wei, L. (2020). Empirical Comparison and Analysis of Web-Based Cell-Penetrating Peptide Prediction Tools. Brief. Bioinform. 21 (2), 408-420. doi:10.1093/bib/bby124

Su, R., Liu, X., Wei, L., and Zou, Q. (2019). Deep-Resp-Forest: A Deep forest Model to Predict Anti-cancer Drug Response. Methods 166, 91-102. doi:10.1016/j. ymeth.2019.02.009

Su, R., Wu, H., Xu, B., Liu, X., and Wei, L. (2019). Developing a Multi-Dose Computational Model for Drug-Induced Hepatotoxicity Prediction Based on Toxicogenomics Data. Ieee/acm Trans. Comput. Biol. Bioinf. 16 (4), 1231-1239. doi:10.1109/tcbb.2018.2858756

Su, W., Liu, M.-L., Yang, Y.-H., Wang, J.-S., Li, S.-H., Lv, H., et al. (2021). PPD: A Manually Curated Database for Experimentally Verified Prokaryotic Promoters. J. Mol. Biol. 433 (11), 166860. doi:10.1016/j.jmb.2021.166860

Szilágyi, A., and Skolnick, J. (2006). Efficient Prediction of Nucleic Acid Binding Function from Low-Resolution Protein Structures. J. Mol. Biol. 358 (3), 922-933. doi:10.1016/j.jmb.2006.02.053

Tang, H., Zhao, Y.-W., Zou, P., Zhang, C.-M., Chen, R., Huang, P., et al. (2018). HBPred: a Tool to Identify Growth Hormone-Binding Proteins. Int. J. Biol. Sci. 14 (8), 957-964. doi:10.7150/ijbs.24174

Tang, Y.-J., Pang, Y.-H., Liu, B., and Idp-Seq2Seq (2020). IDP-Seq2Seq: Identification of Intrinsically Disordered Regions Based on Sequence to Sequence Learning. Bioinformaitcs 36 (21), 5177-5186. doi:10.1093/bioinformatics/btaa667

Tyagi, A., Kapoor, P., Kumar, R., Chaudhary, K., Gautam, A., and Raghava, G. P. S. (2013). In Silico models for Designing and Discovering Novel Anticancer Peptides. Sci. Rep. 3, 2984. doi:10.1038/srep02984

Verkman, A. S. (2005). More Than Just Water Channels: Unexpected Cellular Roles of Aquaporins. J. Cel. Sci. 118 (Pt 15), 3225-3232. doi:10.1242/jcs.02519

Wang, D., and Owler, B. K. (2011). Expression of AQP1 and AQP4 in Paediatric Brain Tumours. J. Clin. Neurosci. 18 (1), 122-127. doi:10.1016/j.jocn.2010.07.115

Wang, H., Ding, Y., Tang, J., and Guo, F. (2020). Identification of Membrane Protein Types via Multivariate Information Fusion with Hilbert-Schmidt Independence Criterion. Neurocomputing 383, 257-269. doi:10.1016/j.neucom.2019.11.103

Warth, A., Simon, P., Capper, D., Goeppert, B., Tabatabai, G., Herzog, H., et al. (2007). Expression Pattern of the Water Channel Aquaporin-4 in Human Gliomas Is Associated with Blood-Brain Barrier Disturbance but Not with Patient Survival. J. Neurosci. Res. 85 (6), 1336-1346. doi:10.1002/jnr.21224

Wei, L., Chen, H., and Su, R. (2018). M6APred-EL: A Sequence-Based Predictor for Identifying N6-Methyladenosine Sites Using Ensemble Learning. Mol. Ther. Nucleic Acids 12, 635-644. doi:10.1016/j.omtn.2018.07.004

Wei, L., Hu, J., Li, F., Song, J., Su, R., and Zou, Q. (2020). Comparative Analysis and Prediction of Quorum-sensing Peptides Using Feature Representation Learning and Machine Learning Algorithms. Brief. Bioinform. 21 (1), 106-119. doi:10.1093/bib/bby107

Wei, L., Liao, M., Gao, Y., Ji, R., He, Z., and Zou, Q. (2014). Improved and Promising Identification of Human MicroRNAs by Incorporating a HighQuality Negative Set. Ieee/acm Trans. Comput. Biol. Bioinf. 11 (1), 192-201. doi:10.1109/tcbb.2013.146

Wei, L., Tang, J., and Zou, Q. (2017). Local-DPP: An Improved DNA-Binding Protein Prediction Method by Exploring Local Evolutionary Information. Inf. Sci. 384, 135-144. doi:10.1016/j.ins.2016.06.026

Wei, L., Wan, S., Guo, J., and Wong, K. K. (2017). A Novel Hierarchical Selective Ensemble Classifier with Bioinformatics Application. Artif. Intelligence Med. 83, 82-90. doi:10.1016/j.artmed.2017.02.005

Wei, L., Xing, P., Zeng, J., Chen, J., Su, R., and Guo, F. (2017). Improved Prediction of Protein-Protein Interactions Using Novel Negative Samples, Features, and an Ensemble Classifier. Artif. Intelligence Med. 83, 67-74. doi:10.1016/j.artmed. 2017.03.001
Wei, L., Zhou, C., Chen, H., Song, J., and Su, R. (2018). ACPred-FL: a SequenceBased Predictor Using Effective Feature Representation to Improve the Prediction of Anti-cancer Peptides. Bioinformatics 34 (23), 4007-4016. doi:10.1093/bioinformatics/bty451

Wu, X., and Yu, L. (2021). EPSOL: Sequence-Based Protein Solubility Prediction Using Multidimensional Embedding. Bioinformatics (Oxford, England) 37, 4314-4320. doi:10.1093/bioinformatics/btab463

Yang, H., Luo, Y., Ren, X., Wu, M., He, X., Peng, B., et al. (2021). Risk Prediction of Diabetes: Big Data Mining with Fusion of Multifarious Physical Examination Indicators. Inf. Fusion 75, 140-149. doi:10.1016/j.inffus.2021.02.015

Yu, L., Wang, M., Yang, Y., Xu, F., Zhang, X., Xie, F., et al. (2021). Predicting Therapeutic Drugs for Hepatocellular Carcinoma Based on Tissue-specific Pathways. Plos Comput. Biol. 17 (2), e1008696. doi:10.1371/journal.pcbi.1008696

Yu, X., Zhou, J., Zhao, M., Yi, C., Duan, Q., Zhou, W., et al. (2021). Exploiting XG Boost for Predicting Enhancer-Promoter Interactions. Cbio 15 (9), 1036-1045. doi:10.2174/1574893615666200120103948

Zeng, X., Liao, Y., Liu, Y., and Zou, Q. (2017). Prediction and Validation of Disease Genes Using HeteSim Scores. Ieee/acm Trans. Comput. Biol. Bioinf. 14 (3), 687-695. doi:10.1109/tcbb.2016.2520947

Zeng, X., Liu, L., Lü, L., and Zou, Q. (2018). Prediction of Potential DiseaseAssociated microRNAs Using Structural Perturbation Method. Bioinformatics 34 (14), 2425-2432. doi:10.1093/bioinformatics/bty112

Zeng, X., Zhu, S., Liu, X., Zhou, Y., Nussinov, R., and Cheng, F. (2019). deepDR: a Network-Based Deep Learning Approach to In Silico Drug Repositioning. Bioinformatics 35 (24), 5191-5198. doi:10.1093/bioinformatics/btz418

Zeng, X., Zhu, S., Lu, W., Liu, Z., Huang, J., Zhou, Y., et al. (2020). Target Identification Among Known Drugs by Deep Learning from Heterogeneous Networks. Chem. Sci. 11 (7), 1775-1797. doi:10.1039/c9sc04336e

Zhang, D., Chen, H.-D., Zulfiqar, H., Yuan, S.-S., Huang, Q.-L., Zhang, Z.-Y., et al. (2021). iBLP: An XGBoost-Based Predictor for Identifying Bioluminescent Proteins. Comput. Math. Methods Med. 2021, 1-15. doi:10.1155/2021/6664362

Zhang, J., Chen, Q., and Liu, B. (2020). iDRBP_MMC: Identifying DNA-Binding Proteins and RNA-Binding Proteins Based on Multi-Label Learning Model and Motif-Based Convolutional Neural Network. J. Mol. Biol. 432 (22), 5860-5875. doi:10.1016/j.jmb.2020.09.008

Zhang, L., Xiao, X., and Xu, Z.-C. (2020). iPromoter-5mC: A Novel Fusion Decision Predictor for the Identification of 5-Methylcytosine Sites in Genome-wide DNA Promoters. Front. Cell Dev. Biol. 8, 614. doi:10.3389/fcell.2020.00614

Zhang, Z., Chen, Z., Song, Y., Zhang, P., Hu, J., and Bai, C. (2010). Expression of Aquaporin 5 Increases Proliferation and Metastasis Potential of Lung Cancer. J. Pathol. 221 (2), 210-220. doi:10.1002/path.2702

Zhu, X.-J., Feng, C.-Q., Lai, H.-Y., Chen, W., and Hao, L. (2019). Predicting Protein Structural Classes for Low-Similarity Sequences by Evaluating Different Features. Knowledge-Based Syst. 163, 787-793. doi:10.1016/j.knosys.2018.10.007

Zhu, Y., Li, F., Xiang, D., Akutsu, T., Song, J., and Jia, C. (2021). Computational Identification of Eukaryotic Promoters Based on Cascaded Deep Capsule Neural Networks. Brief Bioinform 22 (4), bbaa299. doi:10.1093/bib/bbaa299

Zou, Q., Lin, G., Jiang, X., Liu, X., and Zeng, X. (2020). Sequence Clustering in Bioinformatics: an Empirical Study. Brief. Bioinform. 21 (1), 1-10. doi:10.1093/ bib/bby090

Conflict of Interest: The authors declare that the research was conducted in the absence of any commercial or financial relationships that could be construed as a potential conflict of interest.

Publisher's Note: All claims expressed in this article are solely those of the authors and do not necessarily represent those of their affiliated organizations, or those of the publisher, the editors and the reviewers. Any product that may be evaluated in this article, or claim that may be made by its manufacturer, is not guaranteed or endorsed by the publisher.

Copyright $\odot 2022$ Chen, Jiao, Zhao, Zou, Xu, Zhang and Su. This is an open-access article distributed under the terms of the Creative Commons Attribution License (CC $B Y)$. The use, distribution or reproduction in other forums is permitted, provided the original author(s) and the copyright owner(s) are credited and that the original publication in this journal is cited, in accordance with accepted academic practice. No use, distribution or reproduction is permitted which does not comply with these terms. 